\title{
Quasi-Optical SIS Mixers with Normal Metal Tuning Structures
}

\author{
Mei Bin, M. C. Gaidis*, J. Zmuidzinas and T. G. Phillips \\ Downs Laboratory of Physics 320-47, California Institute of Technology, Pasadena, CA 91125, USA. \\ H. G. LeDuc \\ Center for Space Microelectronics Technology, Jet Propulsion Laboratory 320-231, Pasadena, CA 91109, USA.
}

\begin{abstract}
We recently reported [8] a quasi-optical SIS mixer which used $\mathrm{Nb} / \mathrm{Al}$-oxide/ $\mathrm{Nb}$ tunnel junctions and a normal-metal (AI) tuning circuit to achieve an uncorrected receiver noise temperature of $840 \mathrm{~K}$ (DSB) at $1042 \mathrm{GHz}$. Here we present results on several different device designs, which together cover the $300-1200 \mathrm{GHz}$ frequency range. The mixers utilize an antireflection-coated silicon hyperhemispherical lens, a twin-slot antenna, and a two-junction tuning circuit. The broad-band frequency response was measured using Fourier transform spectrometry (FTS), and is in good agreement with model calculations. Heterodyne tests were carried out from $400 \mathrm{GHz}$ up to $1040 \mathrm{GHz}$, and these measurements agree well with the FTS results and with calculations based on Tucker's theory.

Keywords-SIS Mixers, Submillimeter Receivers
\end{abstract}

\section{INTRODUCTION}

$\mathrm{T}$ HE theoretical upper frequency limit for SIS mixers is twice the gap frequency, or about $1.4 \mathrm{THz}$ for mixers using $\mathrm{Nb}$-trilayer tunnel junctions [1]-[3]. To date, good results have been obtained up to $800 \mathrm{GHz}$ using all-niobium circuits [4]-[6].

Modern SIS mixers generally use integrated tuning circuits to resonate the junction capacitance. However, since the RF loss of superconductors increases dramatically above the gap frequency, the noise temperatures of all-niobium mixers increase substantially above $700 \mathrm{GHz}$. To reduce this RF loss above the gap frequency, tuning circuits made of a high conductivity normal metal such as aluminum have been fabricated, and encouraging receiver performance has been obtained [7], [8], [9].

In previous papers, we described results for a $1 \mathrm{THz}$ quasi-optical SIS receiver with an aluminum tuning circuit [8], [10]. An uncorrected double-sideband (DSB) receiver noise temperature of $840 \mathrm{~K}$ was obtained at $1042 \mathrm{GHz}$, with the device cooled to $2.5 \mathrm{~K}$. Corrected for the losses and thermal noise from the LO/signal injection beamsplitter, the receiver noise temperatures are $\sim 770 \mathrm{~K}$ from $822 \mathrm{GHz}$

This work was supported in part by grants from NASA (NAGW107 and NAG2-744), NASA/JPL, and a NSF PYI grant to J.Z. The junction fabrication was performed at the Center for Space Microelectronics Technology, Jet Propulsion Laboratory, California Institute of Technology, and was sponsored by NASA, Office of Space Access Technology.

*Present address: Submillimeter-Wave Superconductive Sensor De velopment Group, Jet Propulsion Laboratory 168-314, Californial Institute of Technology, Pasadena, CA 91109, USA.

E-mail: mei@tacos.caltech.edu.
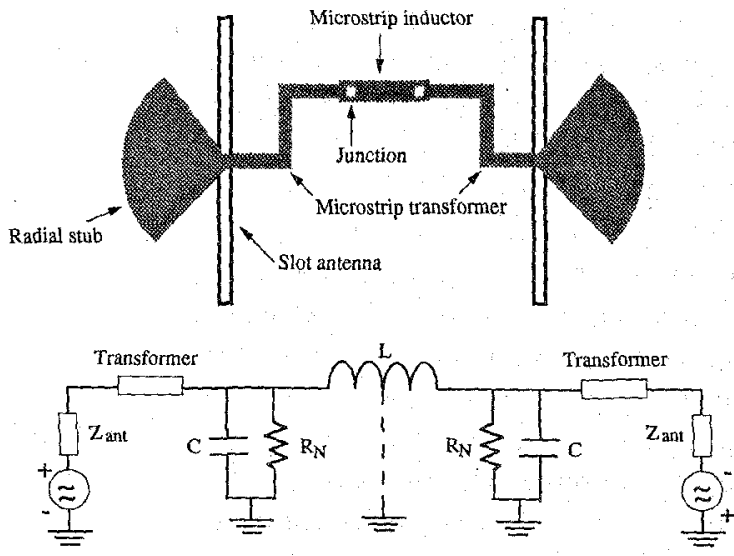

Fig. 1. Schematic layout of the mixer and its equivalent circuit.

to $1042 \mathrm{GHz}$ (at $2.5 \mathrm{~K}$ ). In this paper, we report on the extension of this work to other frequency bands, in an effort to verify our understanding of the device performance. We discuss the design and give test results for Al-wiring mixers optimized for four frequency bands centered at 450,750 , 850 , and $1050 \mathrm{GHz}$. The frequency response in direct detection was obtained using Fourier transform spectroscopy (FTS), and agrees with the predicted response. An analysis of the mixer performance based on Tucker's theory [1] is also presented.

\section{MiXeR DESIGN}

The design details for the planar twin-slot antenna, the two-junction SIS tuning circuit, the mixer block, and the optical layout have been presented elsewhere [5], [11], [12]: Fig. 1 displays the schematic structure representative of the mixers used in this work. The microstrip line between the two junctions serves as the tuning inductor to resonate out the junction capacitances. The bent microstrip lines con necting the junctions to the radial stubs serve as impedance transformers. A virtual ground is formed at the center of the tuning inductor, due to the antisymmetric excitatior from the two slot antennas. Therefore, only half of the circuit needs to be analyzed.

We have written a computer program to simulate anc optimize the mixer circuit. The circuit model include the frequency-dependent impedance of the antennas, mi 
TABLE I

DESIGN PARAMETERS FOR THE TUNING CITCUITS.

\begin{tabular}{ccccc}
\hline$f$ & \multicolumn{2}{c}{$C_{j}=85 \mathrm{fF} / \mu \mathrm{m}^{2}$} & \multicolumn{2}{c}{$C_{j}=65 \mathrm{fF} / \mu \mathrm{m}^{2}$} \\
\cline { 2 - 5 }$(\mathrm{GHz})$ & ind. & transf. & ind. & transf. \\
\hline 450 & $5 \times 46.0$ & $4.5 \times 85.0$ & $5 \times 60.0$ & $4.1 \times 86.0$ \\
750 & $5 \times 15.2$ & $5.0 \times 51.0$ & $5 \times 20.6$ & $4.6 \times 52.5$ \\
850 & $5 \times 11.6$ & $5.0 \times 45.0$ & $5 \times 15.6$ & $4.7 \times 46.0$ \\
1050 & $5 \times 7.4$ & $5.1 \times 36.0$ & $5 \times 10.0$ & $4.8 \times 37.0$ \\
\hline
\end{tabular}

crostrip lines, and tunnel junctions. The properties of the normal-metal Al microstrip lines are calculated using a method which includes the surface impedance of $\mathrm{Al}$ as given by the anomalous skin effect for finite thickness films [10]. The simulation program was used to generate device designs for center frequencies at $450,750,850$, and $1050 \mathrm{GHz}$, by maximizing the RF coupling averaged over a $100 \mathrm{GHz}$ bandwidth. Several devices were designed for each band to allow for parameter variations, particularly in the junction area and specific capacitance. For each band, the tuning circuit was optimized for a single value of the junction area, $1.7 \mu \mathrm{m}^{2}$, but two different values of the specific capacitance were used, $65 \mathrm{fF} / \mu \mathrm{m}^{2}$ and $85 \mathrm{fF} / \mu \mathrm{m}^{2}$. For each tuning circuit design, we included three nominal junction areas $\left(1.2 \mu \mathrm{m}^{2}, 1.7 \mu \mathrm{m}^{2}\right.$, and $2.3 \mu \mathrm{m}^{2}$ ) on the mask layout. Thus there were four bands, eight tuning circuits, and 24 different device designs. For the design, we assumed $R_{n} A=20 \Omega \mu \mathrm{m}^{2}$, which is appropriate for $\mathrm{Nb} / \mathrm{Al}$-oxide/ $\mathrm{Nb}$ junctions with $J_{c} \approx 10 \mathrm{kA} \mathrm{cm}^{-2}, \rho(300 \mathrm{~K})=2.45 \mu \Omega$-cm for aluminum, and $\rho(300 \mathrm{~K}) / \rho(4 \mathrm{~K}) \approx 10$ for the Al films. The measured resistivity ratio was about $5[8]$. The Al films are $2000 \AA$ thick, while two thicknesses of $\mathrm{SiO}\left(\epsilon_{r}=5.6\right)$ were used for the microstrip sections: $2000 \AA$ for the tuning inductor, and $4000 \AA$ for the impedance transformer.

The parameters for the eight tuning circuits are given in Table I. The dimensions of the microstrips are given as $w \times l$ (in $\mu \mathrm{m}$ ). The length $l$ of the inductor is defined as the distance between the centers of the two junctions. All devices use a short $(2.5 \mu \mathrm{m})$ section of microstrip, with the same width as the inductor $(w=5 \mu \mathrm{m})$, to connect the transformer section with the inductor section. The devices were fabricated using a modified $\mathrm{Nb} / \mathrm{Al}$-oxide/ $\mathrm{Nb}$ junction process [8]. No significant lithographic undercut was observed for the batch of devices tested.

The results presented are labeled using three or four digit uumbers that identify the device designs. The first one or iwo digits refers to the frequency band: $4,7,8,10$ correspond to $450,750,850$, and $1050 \mathrm{GHz}$, respectively. The lext digit gives the junction size, with $1,2,3$ referring to $1.2 \mu \mathrm{m}^{2}, 1.7 \mu \mathrm{m}^{2}$, and $2.3 \mu \mathrm{m}^{2}$. The last digit represents ipecific capacitance assumed for the design, with 6 and 8 ndicating $65 \mathrm{fF} / \mu \mathrm{m}^{2}$ and $85 \mathrm{fF} / \mu \mathrm{m}^{2}$.

\section{Receiver Performance}

The devices were measured in a test dewar, whose presure window was either a $25 \mu \mathrm{m}$ thick Mylar sheet or a
$2.2 \mathrm{~mm}$ thick quartz plate, which for some measurements had a Teflon anti-reflection (AR) coating. The $77 \mathrm{~K} \mathrm{IR}$ filter was either an AR-coated quartz plate or a sheet of Zitex film [13]. The mixer optics include a silicon hyperhemispherical lens, with AR-coatings optimized for each of the four frequency bands, and a polyethelene lens mounted on the mixer block to further shape the beam. Our previous paper [5] gives more details on the receiver optics and mixer block construction.

\section{A. Fourier Transform Spectroscopy}

The receiver response as a function of frequency was measured with an FTS system using the SIS junction as a direct detector. A description of the FTS system as well as our measurements for all-niobium circuits can be found in the paper by Gaidis et al. [5]. As in our previous work, we find that the heterodyne noise temperatures correlate well with the direct-detection frequency response measured on the FTS (see Fig. 4).

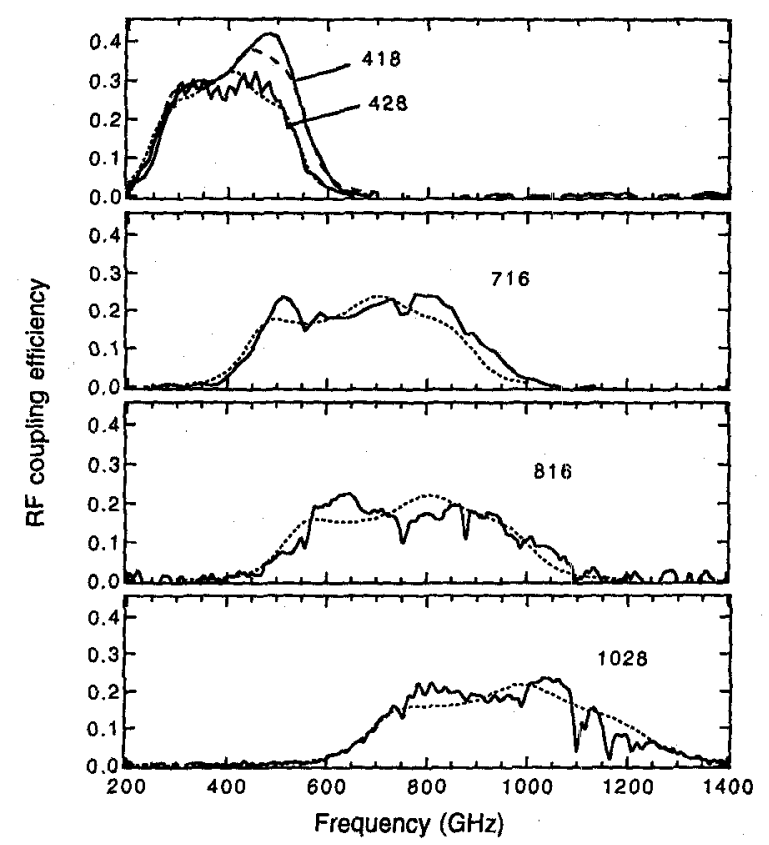

Fig. 2. The response measured on the FTS (solid lines) vs. the calculated RF coupling (dashed lines) for five device designs $(418,428$, 716,816 , and 1028). These devices are all from the same fabrication batch. All the simulations are calculated using a specific capacitance value of $85 \mathrm{fF} / \mu \mathrm{m}^{2}$ and the nominal design values for the rest of the parameters. The aluminum microstrip lines are calculated using the nonlocal theory of the anomalous skin effect, using the measured resistivity ratio of $\rho(300 \mathrm{~K}) / \rho(4 \mathrm{~K})=5$.

Fig. 2 shows a comparison of the theoretical and experimental frequency response measured for devices in each of the four frequency bands. The simulation curves give the RF coupling efficiency, which is the fraction of the power received by the antenna that is dissipated in the tunnel junctions. Since we cannot measure the absolute response, the vertical scaling of the FTS data is adjusted to best match the simulation. In addition, the junction specific capacitance used in the simulations was adjusted to fit the 
shapes of the FTS spectra. There is good agreement between theory and experiment for a specific capacitance of $85 \mathrm{fF} / \mu^{2}$, which is the same value we infer from measurements on all- $\mathrm{Nb}$ circuits [5]. The agreement between simulation and experiment is reasonably good, given that the measurements are affected by water absorption lines at $557,752,986,1094,1114,1163,1211$, and $1229 \mathrm{GHz}$, and also by Fabry-Perot resonances (with a $30 \mathrm{GHz}$ spacing) from the quartz IR filter.

\section{B. Heterodyne Measurements}

We have measured the noise temperatures of several devices, including those in Fig. 2, using the $Y$-factor method with a room temperature hot load and a liquid nitrogen temperature cold load. Fig. 3 displays receiver noise temperatures as a function of frequency for several devices at $4.2 \mathrm{~K}$. The increase in noise temperature with frequency is partly due to the decreasing output power of the LO's. At the highest frequencies, thick Mylar beamsplitters (25 $\mu \mathrm{m}$ and $51 \mu \mathrm{m}$ ) were needed to couple sufficient LO power into the relatively large--area junctions. For the $982 \mathrm{GHz}$ measurement at $4.2 \mathrm{~K}$, which was obtained using a solid-state multiplied $(\times 9)$ Gunn oscillator source [14], the receiver noise temperature drops from $1450 \mathrm{~K}$ to $950 \mathrm{~K}$ after correcting for the loss and thermal noise associated with the beamsplitter. This calculation is supported by the significantly lower uncorrected noise temperature that was measured for same device at $1042 \mathrm{GHz}$. At this frequency, a far-infrared laser was used as the LO source, which produces a good deal more power than the multiplied Gunn LO. Thus, a much thinner beamsplitter $(10 \mu \mathrm{m})$ could be employed.

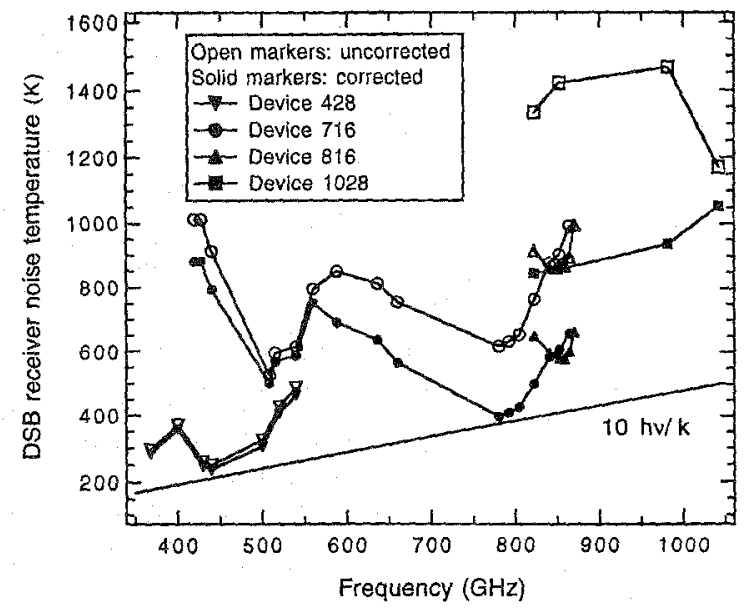

Fig. 3. The DSB noise temperatures of four Al-wiring SIS receivers measured at $4.2 \mathrm{~K}$. The open markers are uncorrected DSB receiver noise temperatures, while the corresponding solid markers include a correction for the LO injection beamsplitter.

The high-frequency devices were also tested at a pumped-LHe temperature of $2.5 \mathrm{~K}$. Upon cooling to $2.5 \mathrm{~K}$, the noise decreases by $100 \mathrm{~K}-300 \mathrm{~K}$ over the band, in part due to the reduced dark current and its associated shot noise. For instance, the $1042 \mathrm{GHz}$ receiver noise drops from $1170 \mathrm{~K}$ to $840 \mathrm{~K}$ upon cooling. This $40 \%$ improvement in the receiver noise temperature can be largely explained by the reduction in the subgap leakage current and the increase in the gap voltage. Tucker theory calculations based on these facts indicate a $20 \%$ drop in the mixer noise temperature and a $35 \%$ reduction in the conversion loss. Therefore, a $25 \%-30 \%$ reduction in the receiver noise temperature can be expected from these effects. The microstrip lines may have a lower loss at $2.5 \mathrm{~K}$ due to a decrease in the $\mathrm{Al}$ resistivity. However, we measured only a $3 \%$ decrease in the AI resistivity. The resulting increase in the $\mathrm{RF}$ coupling efficiency is calculated to be negligible, only $0.4 \%$, and so this effect does not significantly contribute to the decrease of the receiver noise temperature upon cooling.

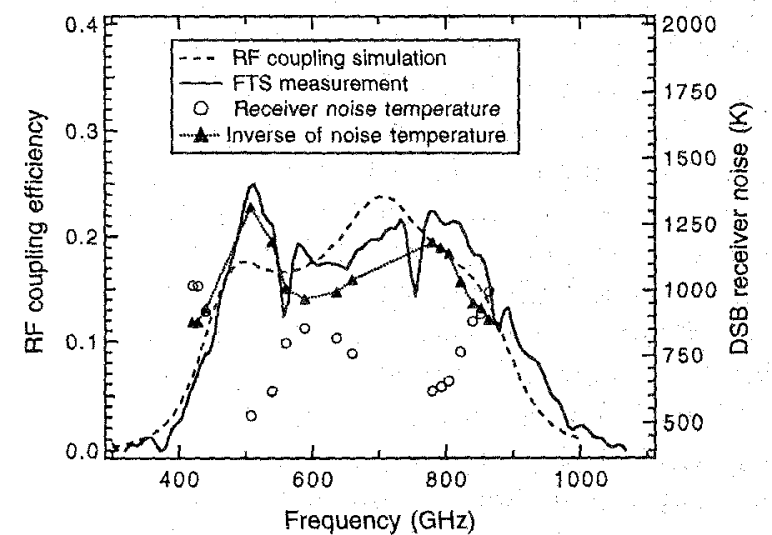

Fig. 4. The FrS spectrum and heterodyne receiver performance for device 716 . The measured and simulated RF coupling efficiencies are plotted vs. frequency. The measured receiver noise temperatures at a bath temperature of $4.2 \mathrm{~K}$ are also shown. Note that the reciprocal of the receiver noise temperature correlates well with the FTS direct detection curve.

In Fig. 4, the receiver noise temperatures measured for device 716 at $4.2 \mathrm{~K}$ are plotted as a function of frequency, along with the FTS spectrum and the calculated frequency response. The broad bandwidth (with a $-3 \mathrm{~dB}$ band of $450-900 \mathrm{GHz}$ ) is typical of the Al-wiring devices, and is a result of the relatively low $Q$-factor of the aluminum microstrip tuning inductance. The reciprocal of the receiver noise temperature is also plotted, multiplied by a scaling factor to allow a comparison with the FTS direct-detection measurement and the circuit calculation. There is clearly a good overall correlation between the heterodyne and di rect detection measurements, and both experimental results agree reasonably well with the calculation. Note that the calculation models only the circuit behavior, and does not include the optical transmission or the antenna bean efficiency. The frequency dependence of these effects could account for some of the discrepancies between the exper. imental and theoretical curves, especially considering the very broad band response of the mixer.

\section{Mixer Performance Analysis}

As an example, we analyze the receiver performancel in terms of the front end optics, the mixer, and the IF ampli 
TABLE II

ESTIMATED TRANSMISSION AND NOISE OF THE OPTICS: DEVICE 1028 AT $982 \mathrm{GHz}$ AND $2.5 \mathrm{~K}$

\begin{tabular}{|c|c|c|c|c|}
\hline \multirow{2}{*}{ Components } & \multirow{2}{*}{$\begin{array}{c}\text { Estimated } \\
\text { transmission } t\end{array}$} & \multicolumn{2}{|c|}{ Temperature (K) } & \multirow{2}{*}{$\begin{array}{c}\text { Noise (K) } \\
T_{\mathrm{n}}=(1-t) T_{\mathrm{eff}}^{\mathrm{anb}} / t\end{array}$} \\
\hline & & $T^{\mathrm{amb}}$ & $T_{\mathrm{eff}}^{\mathrm{amb}}$ & \\
\hline Beamsplitter $25 \mu \mathrm{m} / 51 \mu \mathrm{m}$ & $0.70 / 0.56$ & 295 & 272 & $116.6 / 213.7$ \\
\hline AR-coated qtz. window & 0.91 & 295 & 272 & 26.9 \\
\hline AR-coated qtz. filter & 0.96 & 77 & 55.8 & 2.3 \\
\hline Polyethelene lens & $0 . \overline{94}$ & 2.5 & 0.00 & 0.0 \\
\hline Silicon lens and antenna & $\overline{0.87}$ & 2.5 & 0.00 & 0.0 \\
\hline Al tuning circuit & $\overline{0.23}$ & 2.5 & 0.00 & 0.0 \\
\hline
\end{tabular}

fier chain, for the case of device 1028 at $982 \mathrm{GHz}$ and $2.5 \mathrm{~K}$. At $982 \mathrm{GHz}$, a multiplied Gunn LO source was used which had a nominal output power of $60 \mu \mathrm{W}[14]$; and we were unable to obtain optimum pumping of the junction when using a $25 \mu \mathrm{m}$ thick Mylar beamsplitter. Although using a $51 \mu \mathrm{m}$ thick beamsplitter improved the pumping condition, the (uncorrected) receiver noise temperature increased due to the larger signal loss and thermal noise injection of the thicker beamsplitter.

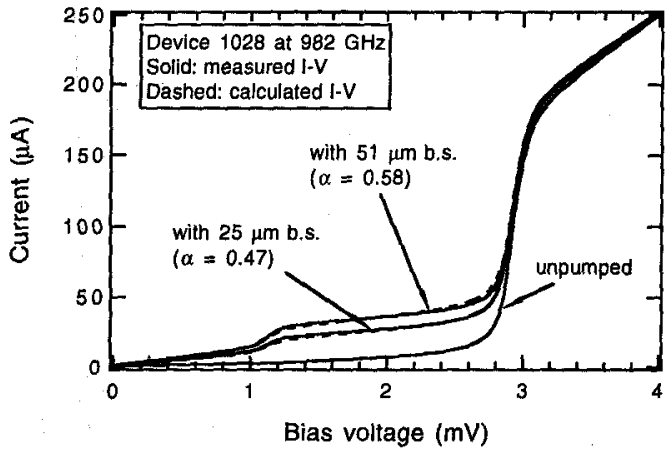

Fig. 5. Unpumped and pumped dc $I-V$ curves for device 1028 at $982 \mathrm{GHz}$ and $2.5 \mathrm{~K}$. The thicker beamsplitter $(51 \mu \mathrm{m})$ couples more LO power onto the junction. The calculated $I-V$ curves for the two pumping cases are also plotted.

To calculate the mixer performance using Tucker's SIS theory in the 3-port approximation [1], the LO pumping strength $\alpha=e V_{\mathrm{LO}} / h \nu$ and the RF embedding admittance $Y_{e}$ seen by the junction must be known. The LO voltage $\alpha$ is easily calculated from the unpumped and pumped $I-V$ curves. With $\alpha=0.47$ for $25 \mu \mathrm{m}$ beamsplitter and $\alpha=0.58$ for $51 \mu \mathrm{m}$ beamsplitter, the calculated $I-V$ curves follow the measured ones very well (see Fig. 5). Since we cannot measure the RF embedding admittance, the value predicted by our circuit program was used: at $982 \mathrm{GHz}$, $Y_{e}=(4.2+j 0.6) G_{n}$, where $G_{n}=(14 \Omega)^{-1}$ is the normalstate conductance of the junction. Using Tucker's theory, the mixer gain $G_{\text {mix }}$ and the mixer noise temperature $T_{\text {mix }}$ are calculated. The theory predicts that the optimum pumping for the mixer would occur around $\alpha=0.7$.

To calculate the receiver performance, we also need to account for the optical losses and thermal noise, the losses (a)
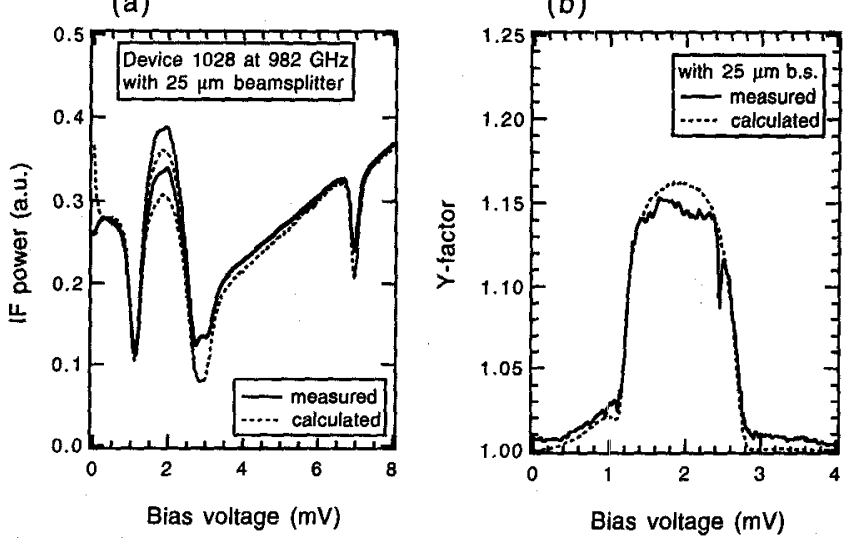

Fig. 6. Measured and calculated IF output power (a) and $\mathrm{Y}$-factor (b) for device 1028 at $982 \mathrm{GHz}$ and $2.5 \mathrm{~K}$ bath temperature. The calculation assumes a LO pumping strength $\alpha=0.47$ (see Fig. 5).

of the aluminum tuning circuit, and the noise of the IF amplifier. The transmission and noise of the optics and tuning circuit are estimated and listed in Table II. The estimates are calculated values and were not adjusted to match the experimental results. In the table, $T^{\mathrm{amb}}$ is the ambient temperature of an optical component, $T_{\mathrm{eff}}^{\mathrm{amb}}$ is the Planck effective temperature, and $T_{\mathrm{n}}$ is the added noise referred to the input of the component [10]. Note the use of a $51 \mu \mathrm{m}$ beamsplitter increases the RF loss by $1 \mathrm{~dB}$ and the RF noise by approximately $100 \mathrm{~K}$, when compared to the use of a $25 \mu \mathrm{m}$ beamsplitter. The shot noise technique [15][17] was used to obtain the IF amplifier noise $T_{\mathrm{IF}}$ and gain $G_{\mathrm{IF}}$. The IF output power $P_{\mathrm{IF}}$ can be calculated according to

$$
P_{\mathrm{IF}}=k_{\mathrm{B}} \Delta \nu\left[\left(T_{\mathrm{eff}}^{\mathrm{sig}}+T_{\mathrm{mix}}\right) G_{\mathrm{mix}}+T_{\mathrm{IF}}\right] G_{\mathrm{IF}},
$$

where $T_{\text {eff }}^{\text {sig }}$ is the Planck effective temperature of the hot or cold load as seen by the junction. In other words, $T_{\text {eff }}^{\text {sig }}$ includes the loss and thermal noise of the front end optics and the RF tuning circuit. For the case of a $25 \mu \mathrm{m}$ beamsplitter, the calculated and measured IF output power vs. bias voltage with hot and cold loads at the receiver input are shown in Fig. 6 . The calculation is only $\sim 5 \%$ lower than the measurement on the first photon step below the gap. The agreement is quite good, given the difficulties 
TABLE III

RECEIVER PERFORMANCE OF DEVICE 1028 AT $982 \mathrm{GHz}$ AND $2.5 \mathrm{~K}$.

\begin{tabular}{|c|c|c|c|c|c|c|c|c|c|}
\hline \multirow{2}{*}{ Beamsplitter } & \multirow{2}{*}{$G_{\mathrm{RF}}$} & \multirow{2}{*}{$T_{\mathrm{RF}}$} & \multicolumn{2}{|c|}{$T_{\mathrm{eff}}^{\mathrm{sig}}$} & \multirow{2}{*}{$\alpha$} & \multirow{2}{*}{$T_{\text {mix }}^{\text {calc }}$} & \multirow{2}{*}{$G_{\text {mix }}^{\text {calc }}$} & \multirow{2}{*}{$T_{\text {rec }}^{\text {calc }}$} & \multirow{2}{*}{$T_{\text {rec }}^{\text {meas }}$} \\
\hline & & & hot & cold & & & & & \\
\hline $25 \mu \mathrm{m}$ & $-9.2 \mathrm{~dB}$ & $158.6 \mathrm{~K}$ & $51.7 \mathrm{~K}$ & $25.8 \mathrm{~K}$ & 0.47 & $87 \mathrm{~K}$ & $-7.4 \mathrm{~dB}$ & $1287 \mathrm{~K}$ & $1339 \mathrm{~K}$ \\
\hline $51 \mu \mathrm{m}$ & $-10.2 \mathrm{~dB}$ & $266.2 \mathrm{~K}$ & $51.7 \mathrm{~K}$ & $29.6 \mathrm{~K}$ & 0.58 & $85 \mathrm{~K}$ & $-6.8 \mathrm{~dB}$ & $1594 \mathrm{~K}$ & $1620 \mathrm{~K}$ \\
\hline
\end{tabular}

TABLE IV

DSB MIXER GONVERSION LOSS AND NOISE TEMPERATURE.

\begin{tabular}{cccccc}
\hline $\begin{array}{c}\text { Device } \\
\text { name }\end{array}$ & $\begin{array}{c}f \\
(\mathrm{GHz})\end{array}$ & $\begin{array}{c}\text { Bath T } \\
(\mathrm{K})\end{array}$ & $\begin{array}{c}\text { Loss } \\
(\mathrm{dB})\end{array}$ & $\begin{array}{c}\text { Noise } \\
(\mathrm{K})\end{array}$ & $\begin{array}{c}h v / k_{\mathrm{B}} \\
(\mathrm{K})\end{array}$ \\
\hline 428 & 430 & 4.2 & 5.2 & 32 & 20.7 \\
716 & 660 & 4.2 & 6.0 & 76 & 31.7 \\
716 & 780 & 4.2 & 8.0 & 105 & 37.5 \\
1028 & 982 & 2.5 & 6.8 & 85 & 47.2 \\
1028 & 1042 & 4.2 & 8.2 & 131 & 50.1 \\
\hline
\end{tabular}

and uncertainties inherent in estimating the optical and circuit losses a priori. Fig. $6(\mathrm{~b})$ shows the similarly good agreement between the measured and calculated $\mathrm{Y}$-factor.

The calculated results are listed in Table III, where we compare the use of the two beamsplitters. Note that when a $51 \mu \mathrm{m}$ beamsplitter is used, the Tucker theory predicts slightly better mixer performance (lower mixer noise $T_{\text {mix }}^{\text {calc }}$ and smaller mixer conversion loss $G_{\text {mix }}^{\text {calc }}$, because the junction is better pumped. However, the receiver performance is worse since the thicker beamsplitter has greater $\mathrm{RF}$ signal loss and thermal noise. If enough $L O$ power were available and a $10 \mu \mathrm{m}$ beamsplitter could be used, the uncorrected receiver noise is calculated to be $\sim 850 \mathrm{~K}$, which is comparable to the result at $1042 \mathrm{GHz}$ with a FIR laser LO.

The same analysis has been carried out for different devices at several frequencies. The calculated DSB mixer conversion losses and noise temperatures are given in Table IV. Note that the calculated mixer noise is on the quantum noise level $\left(h \nu / k_{\mathrm{B}}\right)$ below the gap frequency, and is only several times the quantum level above the gap frequency and into $\mathrm{THz}$ regime. The receiver performance at $1 \mathrm{THz}$ is controlled largely by the loss in the aluminum tuning circuit.

\section{CONCLuSIONS}

We have demonstrated SIS mixers with aluminum tuning circuits at frequencies from $400 \mathrm{GHz}$ to $1 \mathrm{THz}$. Our circuit design program was verified by comparing its predictions with Fourier transform spectrometer measurements. Significant advances in $\mathrm{THz}$ SIS mixers will require tuning circuits with lower losses, which may be possible using high-quality $\mathrm{NbN}$ films.

\section{ACKNOWLEDGMENT}

We are very grateful to $P$. Zimmerman for the loan of his $1 \mathrm{THz}$ multiplier chain, and thank G. Blake and P. Stockman for their help with the FIR laser measurements.

\section{REFERENCES}

[1] J. R. Tucker and M. J. Feldman, "Quantum detection at millimeter wavelengths," Rev. Mod. Phys., vol. 57, no. 4, pp. 1055-1113, 1985.

[2] D. Winkler and T. Claeson, "High Frequency Limits of Superconducting Tunnel Junction Mixers," J. Appl. Phys., vol 62, pp. $4482-4498,1987$.

[3] M. J. Wengler and D. P. Woody, "Quantum Noise in Heterodyne Detection," IEEE J. Quantum Electronics, vol 23, pp. 613-622, 1987.

[4] G. de Lange, C. E. Honingh, J. J. Kuipers, H. H. A. Schaeffer, R. A. Panhuyzen, T. M. Klapwijk, H. Van de Stadt, and M. M. W. $\mathrm{M}$. de Graauw, "Heterodyne Mixing with Nb Tunnel Junctions Above the Gap Frequency," Appl. Phys. Lett., vol 64, pp. 3039$3041,1994$.

[5] M. C. Gaidis, H. G. LeDuc, M. Bin, D. Miller, J. A. Stern, and J. Zmuidzinas, "Characterization of Low-Noise Quasi-Optical SIS Mixers for the Submillimeter Band," IEEE Trans. Microwave Theory. Tech. , vol 44, pp. 1130-1139, 1996.

[6] C. E. Honingh, S. Haas, D. Hottgenroth, K. Jacobs, J. Stutzki, "Fixed Tuned Waveguide Mixers Around $450 \mathrm{GHz}, 670 \mathrm{GHz}$, and $810 \mathrm{GHz}$ for a Dual Channel Receiver," Proc. Seventh Intl. Symp. Space Terahertz Tech., March 12-14, 1996, pp. 63-70, University of Virginia, Charlottesville, Virginia.

[7] H. Van de Stadt, A. Baryshev, P. Dieleman, T. Klapwijk, S. Kovotonyuk, G. de Lange, I. Lapitskaya, J: Mees, R. Panhuzen, G. Prokopenko, and H. Schaeffer, "A $1 \mathrm{THz}$ Nb SIS Heterodyne Mixer with Normal Metal Tuning Structure," Proc. Sixth Intl. Symp. Space Terahertz Tech., March 21-23, 1995, pp. 66-71, Caltech, Pasadena, California.

[8] M. Bin, M. C. Gaidis, J. Zmuidzinas, T. G. Phillips, and H. G, LeDuc, "Low-noise $1 \mathrm{THz}$ niobium superconducting tunnel junction mixers with a normal metal tuning circuit," Appl. Phys. Lett. , vol 68, pp. 1714-1717, 1996.

[9] H. Van de Stadt, A. Baryshev, J. R. Gao, H. Golstein, Th. de Graauw, W. Hulshoff, S. Kovotonyuk, H. Schaeffer, and N. Whyborn, Proc. Seventh Intl. Symp. Space Terahertz Tech., March 12-14, 1996, pp. 536-537, University of Virginia, Charlottesville, Virginia.

[10] M. Bin, M. C. Gaidis, D. Miller, J. Zmuidzinas, T. G. Phillips, and H. G. LeDuc, "Design and characterization of a quasi-optical SIS receiver for the 1 THz band," Proc. Seventh Intl. Symp. Space Terahertz Tech., March 12-14,1996, pp. 549-560, University of Virginia, Charlottesville, Virginia.

[11] J. Zmuidzinas, H.G. LeDuc, J.A. Stern, and S.R. Cypher, "TwoJunction Tuning Circuits for Submillimeter SIS Mixers," IEEE Trans. Microwave Theory Tech, vol 42, pp. 698-706, 1994.

[12] J. Zmuidzinas, N.G. Ugras, D. Miller, M. Gaidis, H.G. LeDuc, and J.A. Stern, "Low-Noise Slot Antenna SIS Mixers" IEEE Trans. Appl. Superconductivity, vol 5, pp. 3053-3056, 1995.

[13] Norton Performance Plastics, 150 Dey Rd., Wayne, NJ 07470.

[14] Radiometer Physics, Bergerwiessen Str. 15, 5309 Meckenheim Germany.

[15] S. Rudner, M. 'J. Feldman, E. Kollberg, and T. Claeson, "Superconductor-insulator-superconductor mixing with arrays at millimeter-wave frequencies," J. Appl. Phys. , vol. 52, pp. 6366, 1981.

[16] D. P. Woody, R. E. Miller, and M. J. Wengler, " $85-115 \mathrm{GHz}$ receivers for radio astronomy," IEEE Trans. Microwave Theory Tech., vol. 33, no. 2, pp. 90-95, 1985.

[17] N. B. Dubash, M. J. Wengler, and J. Zmuidzinas, "Shot nbise and photon-induced correlations in $500 \mathrm{GHz}$ SIS detectors," IEEE Trans. Appl. Supercond., vol. 5, no. 2, pp. 3308-3311, 1995. 